\title{
Early Detection of Cardiotoxicity in Patients Receiving Cancer Chemotherapy Using Three-Dimensional and Speckle Tracking Echocardiography
}

\author{
Hany H. Ebaid, Rizk Sayed R. Sarhan, Ahmed E. Mansour \\ Internal Medicine Department-Cardiology Department, Faculty of Medicine, Benha University, Benha, Egypt \\ ORCID: \\ Hany H. Ebaid: https://orcid.org/0000-0002-5167-8116 \\ Rizk Sayed R Sarhan: https://orcid.org/0000-0002-2069-6661 \\ Ahmed Ezzat Mansour: https://orcid.org/0000-0001-7052-7850
}

\section{Abstract}

Background: Cancer and cardiovascular diseases are prevalent worldwide, especially in the developed world. Chemotherapeutic agents can cause short- and long-term cardiotoxic effects ranging from asymptomatic and transient to clinically significant and long-lasting cardiac events. Using of left ventricular ejection fraction (LVEF) is considered the most important component in the prediction and detection of cardiotoxicity caused by chemotherapy. The drop in LVEF occurs mostly in the last stages, and hence, it is not sensitive in picking early cardiac toxicity. A reduction in global longitudinal strain (GLS) by speckle tracking echocardiography (STE) is proposed to be the earliest indicator of myocardial dysfunction. This study aimed to assess the role of STE and three-dimensional (3D) EF in early detection of cardiotoxicity in patients receiving cardio-toxic chemotherapy. Materials and Methods: This is a cohort prospective study that was carried out in Benha University Hospitals were 99 patients with different malignancies on different chemotherapeutic regimens were recruited aiming to evaluate the role of 3D echocardiography in early detection of cardiotoxicity of chemotherapy, thorough history, examination, and 3D echocardiography was done. Results: In this study, 99 patients were included, with mean of age was 55 years, $30.3 \%$ of patients were males and $66.7 \%$ of patients were female. The median two-dimensional EF was $67.3 \%$, which decreased to $63.0 \%$ at $3 \mathrm{~m}$ follow-up. While 3D EF was $60.25 \%$, which decreased to $56.0 \%$ at $3 \mathrm{~m}$ follow-up. GLS was -22.5 at $0 \mathrm{~m}$ and -20.4 at $3 \mathrm{~m}$ follow-up. GLS reduction $\geq-8.9 \%$ at 3 months follow-up correlated with decreased $3 \mathrm{D}$ EF\% $<53 \%$ with $86.7 \%$ sensitivity and 58\% specificity. Conclusion: in patients receiving cardiotoxic chemotherapy, the reduction in GLS preceded the decrease in EF. Hence, the early detection of this reduction could help to modify chemotherapeutic regimens to avoid irreversible cardiac damage.

Keywords: Cardiotoxicity, chemotherapy, ejection fraction, three-dimensional speckle tracking echocardiography

\section{INTRODUCTION}

The incidence of cancer-related mortality was decreasing worldwide during the past 2-3 decades recently patients who were diagnosed with a malignant disease has improved outcome with better survival, as a result of the development of novel treatments for malignant diseases. ${ }^{[1,2]}$

Cardiotoxicity constitutes subclinical myocardial dysfunction, arrhythmias, pericarditis, coronary vasospasm, significant

Received: 04-08-2020 Revised: 26-08-2020 Accepted: 08-09-2020

Published Online: 27-11-2020

\begin{tabular}{|c|c|}
\hline \multicolumn{2}{|c|}{ Access this article online } \\
\hline Quick Response Code: & $\begin{array}{l}\text { Website: } \\
\text { http://www.ijcva.com }\end{array}$ \\
\hline 4 & $\begin{array}{l}\text { DOI: } \\
\text { 10.4103/IJCA.IJCA_43_20 }\end{array}$ \\
\hline
\end{tabular}

symptomatic heart failure, etc., The extent of cardiotoxicity depends on the type of treatment utilized and the mechanisms of cardiac damage involved. There are two types of cardiotoxicity; type 1 cardiotoxicity is caused by inhibiting topoisomerase IIb in cardiomyocytes and inducing deoxyribonucleic acid

Address for correspondence: Dr. Ahmed Ezzat Mansour, Faculty of Medicine, Benha University, Benha, Egypt. E-mail: ahmedezzat297@gmail.com

This is an open access journal, and articles are distributed under the terms of the Creative Commons Attribution-NonCommercial-ShareAlike 4.0 License, which allows others to remix, tweak, and build upon the work non-commercially, as long as appropriate credit is given and the new creations are licensed under the identical terms.

For reprints contact: WKHLRPMedknow_reprints@wolterskluwer.com

How to cite this article: Ebaid HH, Sarhan RS, Mansour AE. Early detection of cardiotoxicity in patients receiving cancer chemotherapy using three-dimensional and Speckle Tracking Echocardiography. Int J Cardiovasc Acad 2020;6:169-75. 
double-strand breaks and transcription changes, which is permanent and irreversible; whereas type 2 cardiotoxicity is dose-dependent and reversible, caused by the blocking of human epidermal growth factor receptor 2 (HER2), which is expressed on cardiomyocytes in addition to tumor cells. Anthracyclines such as doxorubicin and epirubicin cause type 1 cardiotoxicity and type 2 are caused by trastuzumab. ${ }^{[3]}$

The time point when cardiotoxicity becomes clinically manifest varies substantially; some cancer treatments induce side effects that appear early after exposure and therefore, may adversely affect oncological therapy while others generate cardiac injuries resulting in clinical problems only years later. The prediction of long-term cardiovascular prognosis is frequently challenging because patients with cancer typically receive multiple cancer drugs and sometimes radiation, with the potential for cardiotoxic effects from interactions among the different therapeutic modalities. ${ }^{[4]}$

However one of the leading causes of morbidity and mortality in cancer patients is a cardiac adverse effect of chemotherapeutic agents.

About $60 \%$ of patients will die when they developed heart failure within 2 years of receiving anthracyclines as they can induce progressive cardiac remodeling as a late consequence of earlier myocyte damage, resulting in late cardiomyopathy, while others may cause transient cardiac dysfunction without long-term consequences. ${ }^{[5,6]}$

Hence during the treatment of malignant diseases, we must consider cardiac toxicity and attention for early recognition and management of such complications. ${ }^{[7]}$

Patients receiving anticancer therapies known to be associated with cardiotoxicity should be considered as stage A HF patients (at risk of HF but without structural heart disease or symptoms of HF) ${ }^{[8]}$

While cardiovascular risk factors should be controlled in all patients with cancer, a thorough cardiovascular risk factor assessment is essential before the initiation of anticancer therapies, especially those therapies with known cardiovascular toxicities. A comprehensive evaluation with appropriate initiation of risk reduction strategies may decrease the likelihood of developing cancer-related cardiovascular complications and/or disease. ${ }^{[9]}$

Cardiac toxicity is defined as a reduction of the left ventricular ejection fraction (LVEF) from baseline $55 \%$ by about $5 \%$ in symptomatic and $10 \%$ in asymptomatic patients. ${ }^{[10]}$

This decrease should be confirmed by repeated cardiac imaging done 2-3 weeks after the baseline diagnostic study showing the initial decrease in LVEF. The LVEF decrease may be further categorized as symptomatic or asymptomatic or concerning reversibility. ${ }^{[11]}$

Cardiac toxicity differs according to the type of drug used and the mechanism of cardiac injury. Type 1 is related to anthracyclines with a cumulative dose effect; this agent leads to double-strand deoxyribonucleic acid damage and topoisomerase IIB inhibition in cardiomyocytes. The affection with anthracyclines is permanent and irreversible. ${ }^{[12,13]}$

Type 2 is related to targeted therapy anti-epidermal growth factor receptor 2(HER 2) such as trastuzumab, which is expressed on malignant cells and cardiomyocytes. This leads to the loss of the HER2 survival pathway. The effect of targeted therapy is reversible and not cumulative effect. ${ }^{[14]}$

Echocardiography is considered a common method for monitoring of cardiac toxicity, as it enables the identification of LV dysfunction, valve, and pericardial diseases, especially LV systolic dysfunction. The conventional echo parameters like LVEF, are not sufficiently sensitive for the detection of early changes in cardiac function. ${ }^{[15]}$

The use of LVEF has important limitations like technique-related variability. Furthermore, the reduction in LVEF is often a late phenomenon, with failure to recover systolic function in up to $58 \%$ of patients despite intervention due to late diagnosis. ${ }^{[16,17]}$

The main limitation of two-dimensional (2D) echocardiography is its relatively moderate reproducibility, which can be improved by the use of three-dimensional (3D) echocardiography. The latter is associated with the best day-to-day reproducibility. ${ }^{[17]}$

However remains dependent on image quality, availability, and operator experience. For serial evaluation of patients with cancer, LVEF measurements should ideally be performed by the same observer with the same equipment to reduce variability. ${ }^{[11]}$

Newer echocardiographic techniques such as 3D echocardiography and speckle tracking echocardiography (STE)-derived global longitudinal strain (GLS) imaging is better used for the early detection of subclinical LV systolic dysfunction. ${ }^{[18]}$

Global systolic longitudinal myocardial strain (GLS) has been reported to accurately predict a subsequent decrease in LVEF. A relative percentage reduction of GLS of. $15 \%$ from baseline is considered abnormal and a marker of early LV subclinical dysfunction. ${ }^{[19]}$

Until the standardization of strain imaging through different vendors is fully achieved, the current recommendation is to use the same equipment for the longitudinal follow-up of patients with cancer to facilitate the interpretation of the results. ${ }^{[2]}$

Normal ranges for GLS are defined as mean GLS of - 19.7\% and a $95 \%$ confidence interval of $-20.4 \%$ to $-18.9 \%$. The diagnosis of cardiotoxicity in both asymptomatic and symptomatic LV dysfunction patients is established by early fall in GLS by STE between $10 \%$ and $15 \%{ }^{[10]}$

\section{Aim of the study}

This study is aimed to assess the role of 3D and STE in the early detection of cardiotoxicity in patients receiving different chemotherapeutic regimens. 


\section{Materials and Methods}

\section{Study design}

This cohort prospective study was conducted at the Hemato-oncology unit, Internal Medicine Department, and Cardiovascular Department, from May 2018 to May 2020, and it was approved by the ethical committee of our University, and written consents were obtained from the included patients. After informing the patients about the nature of the study and written consent was taken, 99 patients were included after fulfilling the following inclusion criteria: patients with newly-diagnosed various types of malignancies for which cardiotoxic chemotherapeutic agents were planned to be prescribed in their treatment regimens, both males and females, and adult patients aged $>18$. Exclusion criteria included hypertensive patients, patients with rheumatic, congenital, or ischemic heart diseases, diabetic patients, patients with other endocrinal, metabolic, or systemic diseases that have cardiac effects, patients receiving any other cardiotoxic therapeutic agents, smokers, and alcoholics.

The patients were subjected to full medical history and thorough clinical examination at Hemato-oncology Unit, Internal Medicine for proper selection of included patients. Venous blood samples were obtained from each patient for performing routine laboratory investigations, including complete blood count, erythrocyte sedimentation rate, liver function tests, renal function tests, fasting, and postprandial blood sugars, glycosylated hemoglobin, and blood lipids profiles. Tumors markers, imaging studies, and tissue biopsies were performed to determine the size, type, and stage of malignancy, and the appropriate chemotherapeutic regimens were defined. Then, the patients were sent to the cardiology department where electrocardiogram (ECG) and echocardiography were done at time 0 before the beginning of the chemotherapy regimen. The patients then return to Hemato-Oncology Unit, Internal medicine Department, for receiving their chemotherapeutic regimens. After completing 3 months of chemotherapy regimens, the included patients were sent again to the Cardiology Department for re-evaluation by ECG and echocardiography. The results of 2D, 3D, and GLS were compared using Philips EPIQ 7 device; the GLS was measured in standard apical two-, three-, and four-chamber views, and aortic valve closure is used for timing of end-systole.

\section{Echocardiographic methods}

All patients underwent standard Doppler echocardiographic examination using M5Sc 2D transducer with the harmonic capability and a 3D volumetric transducer $(4 \mathrm{~V})$ for real-time echocardiographic dataset acquisition of the left ventricle by a VividE9 XDclear ultrasound machine (GE Healthcare, Norway).

All 2D and 3D echocardiography were done by the single operator on the same machine to avoid inter-observer and inter-device variability and discrepancies in the techniques. Intra-observer variability is $<6 \%$ for global longitudinal and circumferential strain, and $<9 \%$ for global transverse and radial strain in $2 \mathrm{D}$ strain imaging and relative mean errors of $4.9 \%-7.3 \%$ for $3 \mathrm{D} \cdot .^{[20,21]}$
A standard 2D echocardiographic examination was done, and the quantitative analysis of the left ventricle was performed according to the recommendations. 2D EF was derived from LV end-diastolic volume and end-systolic volume, which was calculated according to the modified Simpson rule in apical four- and two-chamber views. ${ }^{[22]}$

2D and 3D STE was performed on the same machine and were analyzed as per standard protocols..$^{[22]}$

We calculated 2D GLS as the average of 18 myocardial segments which were recorded in three apical views. ${ }^{[22]}$

\section{Statistical analysis}

Statistical analyses were performed using SPSS (Statistical Package of Social Science) version 20 (SPSS Inc., Chicago, Illinois, USA). Quantitative data were expressed in numbers, range, mean, and standard deviation. Qualitative data were expressed in frequencies and percentages. The Student's $t$-test was used for comparison of mean values of two groups of quantitative data. Value of $P<0.05(P<0.05)$ was considered statistically significant.

\section{REsULTS}

The current study included 99 patients, their mean age was 55 years (interquartile range [IQR], 44-60 years), of them $30(30.3 \%)$ were male. The most common malignancy was cancer breast $(n=40,40.4 \%)$, followed by non-Hodgkin's lymphoma $(n=24,24.2 \%)$, then ovarian cancer $(n=12,12.1 \%)$ [Table 1]. The most common chemotherapeutic regimen used was Adriamycin, Cyclophosphamide, and Taxol (AC/T) $(n=32,32.3 \%)$ followed by Cyclophosphamide, Hydroxydaunorubicin, Oncovin, and Prednisone (CHOP) $(n=24,24.2 \%)$ [Table 1].

Median 2D EF\% was $67.3 \%(63.23-68.2)$ at 0 month which decreased to $63.0 \%(59.0-64.45)$ at 3 months follow-up. 3D EF\% was $60.25 \%(56.13-61.0)$ and $56.0 \%(52.08-57.5)$ at 0 and 3 months, respectively. GLS was $-22.5(-20.25:-22.8)$ at 0 month and $-20.4(-18.43:-20.88)$ at 3 months follow-up [Tables 1 and 2].

There was a significant effect of gender on the GLS, as there were lower values seen in males patients when compared to female patients at both baseline and 3 months. Median GLS at 0 months for males and females was $-18.9:-20.25 \%$ and $-22.5:-22.9 \%$, respectively. While median GLS at 3 months for males and females was -16.9 : $-18.5 \%$ and $-20.35:-20.95 \%$, respectively, and this was statistically significant $P<0.001$ [Tables 3 and 4].

Of the studied patients, 23 (23.2\%) patients had a significant reduction in the GLS at 3 months follow-up (10.27\%), where their GLS decreased from - 20.9 (-18.9:-22.5) to -18.7 (-16.9:-20.1), [Table 5].

The GLS reduction cutoff value was 9.7 at 0 months with sensitivity $100 \%$ and specificity $70.8 \%$, PPV was 9.7 , and the NPV was 100 [Table 6]. 
The GLS reduction $\geq-8.9 \%$ at 3 months follow-up correlates with decreased 3D EF\% $<53 \%$ with sensitivity $86.7 \%$ and specificity $58 \%$ [Table 7].

\section{Discussion}

Cardiotoxicity as an adverse event of chemotherapy includes a range of cardiac problems, including heart failure, myocardial ischemia or infarction, hypertension, thromboembolism, and arrhythmias. ${ }^{[23]}$

The extent of cardiotoxicity depends uon many factors relating to the chemotherapy used as well as patients, such as type of

\section{Table 1: Data of the studied patients}

\begin{tabular}{|c|c|}
\hline & Value $(n=99)$ \\
\hline Age, median (IQR) & $55.0(44.0-60.0)$ \\
\hline \multicolumn{2}{|l|}{ Gender, $n(\%)$} \\
\hline Male & $30(30.3)$ \\
\hline Female & $69(69.7)$ \\
\hline \multicolumn{2}{|l|}{ Regimen, $n(\%)$} \\
\hline A & $20(20.2)$ \\
\hline ABVD & $9(9.1)$ \\
\hline $\mathrm{AC} / \mathrm{T}$ & $32(32.3)$ \\
\hline $\mathrm{CHOP}$ & $24(24.2)$ \\
\hline FAC & $8(8.1)$ \\
\hline MAVC & $6(6.1)$ \\
\hline \multicolumn{2}{|l|}{ Cancer type, $n(\%)$} \\
\hline Cancer bladder & $6(6.1)$ \\
\hline Breast cancer & $40(40.4)$ \\
\hline HL & $9(9.1)$ \\
\hline NHL & $24(24.2)$ \\
\hline Ovarian cancer & $12(12.1)$ \\
\hline STS & $8(8.1)$ \\
\hline Her2, $n(\%)$ & $8(8.1)$ \\
\hline 2D EF at 0 month, median (IQR) & $67.3(63.23-68.2)$ \\
\hline 2D EF at 3 months, median (IQR) & $63.0(59.0-64.45)$ \\
\hline $3 \mathrm{D} \mathrm{EF}$ at 0 month, median (IQR) & $60.25(56.13-61.0)$ \\
\hline 3D EF at 3 months, median (IQR) & $56.0(52.08-57.5)$ \\
\hline GLS at 0 month, median (IQR) & $-22.5(-20.25:-22.8)$ \\
\hline GLS at 3 months, median (IQR) & $-20.4(-18.43:-20.88)$ \\
\hline
\end{tabular}

A: Adriamycin, ABVD: Adriamycin-bleomycin-vinblastine-Dacarbazine, AC/T: Adriamycin-cyclophosphamide/Taxol,

CHOP: Cyclophosphamide -doxorubicin- vincristine -prednisone, FAC: Fluorouracil-Adriamycin-Cytoxan, MAVC: MethotrexateAdriamycin-vinblastine-cisplatin, HL: Hodgkin lymphoma, NHL: NonHodgkin lymphoma, STS: Sarcoma soft tissue, Her2: Human epidermal growth factor receptor 2, 2D EF: 2 dimensional ejection fraction, 3DEF: 3 Dimensional ejection fraction, GLS: Global longitudinal strain, IQR: Interquartile range drug, the dose administered during each cycle, cumulative dose, schedule of administration, route of administration, a combination of other cardiotoxic drugs, or association with radiotherapy, preexisting heart disease, history of hypertension, and age $>65$ years. ${ }^{[24]}$

Strategies for screening and detection of cardiotoxicity include cardiac imaging (echocardiography, nuclear imaging, Cardiac magnetic resonance (CMR), and biomarkers (troponin, natriuretic peptides). The choice of modalities depends upon local expertise and availability, and several important core principles should be considered. ${ }^{[25]}$

In the current study, the diagnostic value of conventional standard 2D echocardiography, 2D-GLS, and 3D-derived strain parameters was evaluated for early detection of LV function in cancer patients who received different chemotherapeutic agents.

In our study, the mean age of our patients was 55 years with IQR (44-60 years) and female patients account for $66.7 \%$ of the total number of studied patients. Moreover, this was concordant with Motoki et al., who studied cardiotoxic effects of anthracyclines on 25 cancer patients, and found that the mean age of patients was $58 \pm 11$ years and female patients account for $56 \%$ of the studied patients. ${ }^{[26]}$ Furthermore, this was in agreement with Cadeddu et al., who studied the possible role of telmisartan in preventing myocardial damage induced by epirubicin on 49 patients free from cardiovascular diseases affected by a variety of solid cancers, and found that the mean age of patients in the study was $56 \pm 13$ years and female patients represent $76 \%$ of patients ${ }^{[27]}$ also, this was supported by the study of Baratta et al., who studied 31 patients of epirubicin treated cancer patients intending to detect early preclinical changes that are predictive of the risk for heart failure, found that the patient's mean age was $59 \pm 14$ years and female patients represent $74 \%$ of the total studied patients. Moreover, this could be explained by increase the incidence of cancer with age and high prevalence of malignancy in females, especially breast cancer. ${ }^{[28]}$

In our study, the most common chemotherapeutic regimen used was AC/T $(n=32,32.3 \%)$ followed by CHOP $(n=24,24.2 \%)$. This was in agreement with Baratta et al., who studied 36 patients with a neoplastic disease with normal myocardial mass and $\mathrm{LVEF} \geq 55 \%$ receiving chemotherapy treatment and showed that the most common drugs used were doxorubicin in $58 \%$ and trastuzumab in $22 \%$ subjects. ${ }^{[29]}$ Furthermore, this was concordant with Stoodley et al. who studied 52 women

Table 2: Comparison between 0 month and 3 months 2 dimensional ejection fraction \%, 3 dimensional ejection fraction $\%$, and global longitudinal strain

\begin{tabular}{|c|c|c|c|c|}
\hline & 0 month & 3 months & Statistical test (Z) & $P$ \\
\hline 2D EF, median (IQR) & $67.3(63.23-68.2)$ & $63.0(59.0-64.45)$ & 8.64 & $<0.001 * *$ \\
\hline 3D EF, median (IQR) & $60.25(56.13-61.0)$ & $56.0(52.08-57.5)$ & 8.66 & $<0.001 * *$ \\
\hline GLS, median (IQR) & $-22.5(-20.25--22.8)$ & $-20.4(-18.43--20.88)$ & 8.67 & $<0.001 * *$ \\
\hline
\end{tabular}

GLS: Global longitudinal strain, IQR: Interquartile range, 2D EF: 2 dimensional ejection fraction, 3DEF: 3 Dimensional ejection fraction. **Highly significant 
Table 3: Comparison between 0 month 2 dimensional ejection fraction \%, 3 dimensional ejection fraction \%, and global longitudinal strain

\begin{tabular}{|c|c|c|c|c|}
\hline At 0 month & Male (30) & Female (69) & Statistical test (Z) & $P$ \\
\hline 2D EF, median (IQR) & $61.4(60.0-63.2)$ & $68.0(66.9-68.5)$ & 7.82 & $<0.001 * *$ \\
\hline 3D EF, median (IQR) & $55.0(54.0-56.13)$ & $60.8(60.0-61.5)$ & 7.72 & $<0.001 * *$ \\
\hline GLS, median (IQR) & $-19.5(-18.9--20.25)$ & $-22.7(-22.5--22.9)$ & 7.79 & $<0.001 * *$ \\
\hline
\end{tabular}

GLS: Global longitudinal strain, IQR: Interquartile range, 2D EF: 2 dimensional ejection fraction, 3DEF: 3 Dimensional ejection fraction. **Highly significant

Table 4: Comparison between 3 months 2 dimensional ejection fraction \%, 3 dimensional ejection fraction \%, and global longitudinal strain

\begin{tabular}{|c|c|c|c|c|}
\hline At 3 months & Male (30) & Female (69) & Statistical test (Z) & $P$ \\
\hline 2D EF, median (IQR) & $58.0(57.0-59.0)$ & $64.0(63.0-65.0)$ & 7.75 & $<0.001 * *$ \\
\hline 3D EF, median (IQR) & $51.5(51.0-52.13)$ & $57.0(56.0-58.0)$ & 7.58 & $<0.001^{* *}$ \\
\hline GLS, median (IQR) & $-17.6(-16.9--18.5)$ & $-20.7(-20.35--20.95)$ & 7.75 & $<0.001 * *$ \\
\hline
\end{tabular}

GLS: Global longitudinal strain, IQR: Interquartile range, 2D EF: 2 dimensional ejection fraction, 3DEF: 3 Dimensional ejection fraction. **Highly significant

\begin{tabular}{lcccc}
\hline \multicolumn{5}{l}{ Table 5: Percentage reduction in global longitudinal strain at $\mathbf{3}$ months compared to $\mathbf{0}$ month } \\
\hline Significant GLS fall & Yes ( $\mathbf{2} \mathbf{1 0} \%)(\mathbf{2 3})$ & No $(<\mathbf{1 0} \%)(\mathbf{7 6})$ & Statistical test $(\mathbf{Z})$ & $\boldsymbol{P}$ \\
\hline GLS 0M, median (IQR) & $20.9(18.9-22.5)$ & $22.6(21.5-22.9)$ & 2.91 & $0.004^{* *}$ \\
GLS 3M, median (IQR) & $18.7(16.9-20.1)$ & $20.6(19.6-20.9)$ & 4.05 & $<0.001^{* *}$ \\
\% GLS reduction, median (IQR) & $10.27(10.15-10.87)$ & $8.84(8.27-9.38)$ & 7.24 & $<0.001^{* *}$ \\
\hline GLS: Global longitudinal strain, IQR: Interquartile range, 2D EF: 2 dimensional ejection fraction, 3DEF: 3 Dimensional ejection fraction. **Highly significant
\end{tabular}

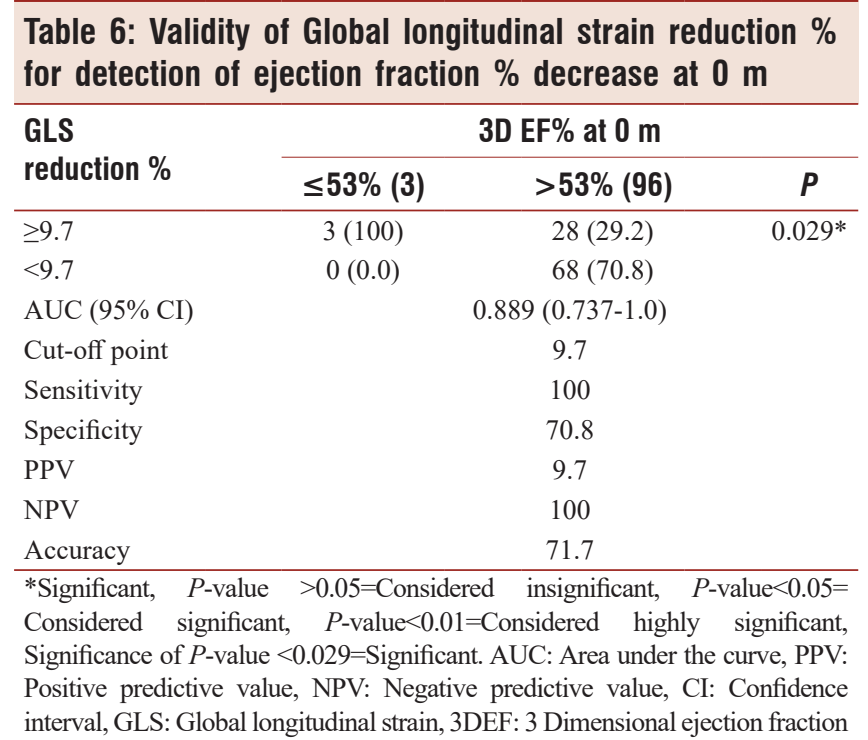

with confirmed breast cancer, had doxorubicin used in $77 \%$ of the studied patients, and epirubicin was used in $23 \%$ of the studied subjects. ${ }^{[30]}$

In this study, we measured the GLS as a method for early detection of cardiotoxicity as a noninvasive, available tool that could be used in our institute to prevent advanced cardiovascular damage with cancer-treated patients.

In this study, we measured the GLS as a method of early detection of cardiotoxicity, and this was concordant with the recent published ESMO consensus recommendations 2020 also this was concordant with the ESC position paper 2016.
The pre-and post-chemotherapy GLS at 0 months and 3 months were - $22.5(-20.25:-22.8)$ and - $20.4(-18.43:-20.88)$, respectively. This was supported by Baratta et al. who stated that pre- and post-chemotherapy, the GLS at 0 months was $\left(-20.3 \% \pm 2.7 \%\right.$, ) and 3 months $(-18.9 \pm 2.5 \%),{ }^{[18]}$ also this was in agreement with Sawaya et al., who studied 81 women with newly diagnosed breast cancer that were treated with anthracyclines followed by taxanes and trastuzumab, showed that pre- and post-chemotherapy the GLS at 0 months $(-21 \% \pm 2 \%)$ and 3 months $(-19.2 \% \pm 2 \%){ }^{[20]}$

The reduction of GLS in the studied patients was $8.67 \%$ from baseline at 3 months. While the percentage reduction was $10.27 \%$ in 23 of the studied subjects $(23.2 \%)$, who had a significant reduction in the GLS at 3 months when compared to the baseline. This was concordant with Negishi et al. who studied 81 female patients with (mean age, $50 \pm 11$ years) receiving trastuzumab, and this study proposed that the GLS at 6 months is considered to be the strongest predictor of chemotherapy-related cardiac toxicity. They concluded that reduction of GLS $<8 \%$ compared with the baseline appears not to be clinically meaningful, whereas $>15 \%$ reduction appears to be of clinical significance. ${ }^{[31]}$ Furthermore, this was concordant with the 2016 ESC position paper on cancer treatments and cardiovascular toxicity, which stated that $>15 \%$ relative percentage reduction of GLS from baseline may suggest the risk of cardiotoxicity. ${ }^{[25]}$

In this study, we found that reductions in myocardial deformation parameters such as GLS are a sign of subclinical myocardial changes from cancer therapy and occur before 


\begin{tabular}{|c|c|c|c|}
\hline \multirow{2}{*}{$\begin{array}{l}\text { GLS reduction } \\
\%\end{array}$} & \multicolumn{3}{|c|}{ 3D EF\% at 3 months } \\
\hline & $\leq 53 \%(30)$ & $>53 \%(69)$ & $P$ \\
\hline$\geq 8.9$ & $26(86.7)$ & $29(42.0)$ & $<0.001 * *$ \\
\hline$<8.9$ & $4(13.3)$ & $40(58.0)$ & \\
\hline AUC $(95 \% \mathrm{CI})$ & & $0.77(0.665-874)$ & \\
\hline Cut-off point & & 8.9 & \\
\hline Sensitivity & & 86.7 & \\
\hline Specificity & & 58.0 & \\
\hline PPV & & 47.3 & \\
\hline NPV & & 90.9 & \\
\hline Accuracy & & 66.7 & \\
\hline
\end{tabular}

AUC: Area under the curve, PPV: Positive predictive value, NPV: Negative predictive value, CI: Confidence interval, GLS: Global longitudinal strain, 3DEF: 3 Dimensional ejection fraction. **Highly significant

any change in LVEF as assessed by conventional 2DE and this was concordant with Thavendiranathan et al. who found the same results. ${ }^{[32]}$

In this study, the median normal GLS at baseline was lower in males when compared to females with values being $-19.5(-18.9:-20.25)$ and $-22.7(-22.5:-22.9)$, respectively. This was supported by the study of Kocabay et al., who reported that a mean GLS was $-20.7 \% \pm 2 \%$ for men and $-22.1 \% \pm 1.8 \%$ for women, respectively. ${ }^{[33]}$

These values were comparable with our study results. Furthermore, this also was in agreement with the Japanese Ultrasound Speckle Tracking of the Left Ventricle study that showed lower GLS values for men when compared to women. ${ }^{[34]}$

In our study, we found that 3D echocardiography is an ideal method for early detection of cardiotoxicity, and this was concordant with recent ESMO consensus recommendations, which stated that 3D echocardiography should be used instead of Quantitative 2D echocardiography using Simpson's biplane method and also better than Cardiac magnetic resonance (CMR) imaging and multigated acquisition (MUGA) as it provides substantial additional information on cardiac structure, valve function, hemodynamics and physiology not typically found with MUGA scanning ${ }^{[35]}$ also, this was concordant with ESC position paper 2016 and EVACI 2017 which recommend the use of 3D echocardiography as the standard method and use $2 \mathrm{D}$ echocardiography only when $3 \mathrm{D}$ is not available. ${ }^{[25]}$

In our study, we found that standard 2D echocardiographic examination, 2D-derived EF is not adequately useful in detecting the early changes in LV function and it could be detected early by $3 \mathrm{D}$ assessment for EF helps in identifying the EF alterations not identified on standard 2D EF quantification techniques and this was concordant with Alam et al. who found the same results and this could be explained by the relatively moderate reproducibility of the 2D echocardiography while $3 \mathrm{D}$ provides the best day-to-day reproducibility. ${ }^{[36]}$

\section{Study limitations}

Limitations of the current study included the small sample size and the relatively short follow-up period, which was reflected in evaluating the early detection of signs of subclinical cardiac affection by 3D echocardiography. Cardiac biomarkers, such as troponins and $\mathrm{N}$-terminal pro-brain-type natriuretic peptide, which could detect early subclinical LV dysfunction were not evaluated in this study.

\section{Conclusion}

Myocardial deformation indices such as GLS are considered an excellent parameter to be used for early detection of subclinical LV dysfunction in cancer chemotherapy subjects. GLS is favored because of a lack of angle dependency and better reproducibility. Early decline in GLS helps physicians to detect subjects who could benefit from cardioprotective therapy and modification of the chemotherapeutic regimen to avoid the development of overt heart failure.

\section{Financial support and sponsorship}

Nil.

\section{Conflicts of interest}

There are no conflicts of interest.

\section{ReFERENCES}

1. International Agency for Research on Cancer. World1 Cancer Fact Sheet. Geneva, Switzerland: World Health Organization; 2012. Available from: http://www.gicr.iarc.fr/files/resources/20120906WorldCancerF actSheet.pdf. [Last accessed on 2013 Sep 01].

2. DeSantis CE, Lin CC, Mariotto AB, Siegel RL, Stein KD, Kramer JL, et al. Cancer treatment and survivorship statistics, 2014. CA Cancer J Clin 2014;64:252-71.

3. Wang $\mathrm{CL}$, Chu PH. Echocardiography for evaluation of oncology therapy-related cardiotoxicity. Acta Cardiol Sin 2016;32:560-4.

4. Khouri MG, Douglas PS, Mackey JR, Martin M, Scott JM, Scherrer-Crosbie M, et al. Cancer therapy-induced cardiac toxicity in early breast cancer: addressing the unresolved issues. Circulation 2012;126:2749-63.

5. Hooning MJ, Botma A, Aleman BM, Baaijens MH, Bartelink $\mathrm{H}$, Klijn JG, et al. Long-term risk of cardiovascular disease in 10-year survivors of breast cancer. J Natl Cancer Inst 2007;99:365-75.

6. Felker GM, Thompson RE, Hare JM, Hruban RH, Clemetson DE, Howard DL, et al. Underlying causes and long-term survival in patients with initially unexplained cardiomyopathy. N Engl J Med 2000;342:1077-84.

7. Negishi K, Negishi T, Haluska BA, Hare JL, Plana JC, Marwick TH. Use of speckle strain to assess left ventricular responses to cardiotoxic chemotherapy and cardioprotection. Eur Heart J Cardiovasc Imaging 2014; 15:324-31.

8. Hunt SA, Baker DW, Chin MH, Cinquegrani MP, Feldman AM, Francis GS, et al. ACC/AHA guidelines for the evaluation and management of chronic heart failure in the adult: executive summary. A report of the American College of Cardiology/American Heart Association Task Force on Practice Guidelines (Committee to revise the 1995 Guidelines for the Evaluation and Management of Heart Failure). J Am Coll Cardiol 2001;38:2101-13.

9. Mehta LS, Watson KE, Barac A, Beckie TM, Bittner V, Dent S, et al. Cardiovascular disease and breast cancer: Where these entities intersect: A scientific statement from the American Heart Association. Circulation 2018;137:e30ee66.

10. Hortobagyi GN, Stemmer SM, Burris HA, Yap YS, Sonke GS, Paluch-Shimon S, et al. Ribociclib as first-line therapy for HR-positive, 
advanced breast cancer. N Engl J Med 2016;375:1738-48.

11. Plana JC, Galderisi M, Barac A, Ewer MS, Bonnie KY, Scherrer-Crosbie M, et al. Expert consensus for multimodality imaging evaluation of adult patients during and after cancer therapy: A report from the American Society of Echocardiography and the European Association of Cardiovascular Imaging. Eur Heart $\mathrm{J}$ Cardiovasc Imaging 2014;15:1063-93.

12. Seidman A, Hudis C, Pierri MK, Shak S, Paton V, Ashby M, et al. Cardiac dysfunction in the trastuzumab clinical trials experience. J Clin Oncol 2002;20:1215-21.

13. Liu KL, Chen JS, Chen SC, Chu PH. Cardiovascular toxicity of molecular targeted therapy in cancer patients: A double-edged sword. Acta Cardiol Sin 2013;29:295-303.

14. Plana JC, Galderisi M, Barac A, Ewer M, Ganame J, Sebag I, et al. 2014. Expert consensus for multimodality imaging evaluation of adult patients during and after cancer therapy: a report from The American Society of Echocardiography and The European Association of Cardiovascular Imaging. J Am Soc Echocardiogr 2014;27:911-39.

15. Bria E, Cuppone F, Milella M, Verma S, Carlini P, Nisticò C, et al. Trastuzumab cardiotoxicity: Biological hypotheses and clinical open issues. Expert Opin Biol Ther 2008;8:1963-71.

16. Zamorano JL, Lancellotti P, Rodriguez Muñoz D, Aboyans V, Asteggiano R, Galderisi M, et al. 2016 ESC Position Paper on cancer treatments and cardiovascular toxicity developed under the auspices of the ESC Committee for Practice Guidelines: The task force for cancer treatments and cardiovascular toxicity of the European Society of Cardiology (ESC). Eur Heart J 2016;37:2768-801.

17. Thavendiranathan P, Grant AD, Negishi T, Plana JC, Popović ZB, Marwick TH. Reproducibility of echocardiographic techniques for sequential assessment of left ventricular ejection fraction and volumes: Application to patients undergoing cancer chemotherapy. J Am Coll Cardiol 2013;61:77-84.

18. Sawaya H, Sebag IA, Plana JC, Januzzi JL, Bonnie KY, Cohen V, et al. Early detection and prediction of cardiotoxicity in chemotherapy-treated patients. Am J Cardiol 2011;107:1375-80.

19. Negishi K, Negishi T, Hare JL, Haluska BA, Plana JC, Marwick TH. Independent and incremental value of deformation indices for prediction of trastuzumab-induced cardiotoxicity. J Am Soc Echocardiogr 2013;26:493-8.

20. Cheng S, Larson MG, McCabe EL, Osypiuk E, Lehman BT, Stanchev P, et al. Reproducibility of speckle-tracking-based strain measures of left ventricular function in a community-based study. J Am Soc Echocardiogr 2013;26:1258-6600.

21. Baysan O, Ocaklı EP, Saglam Y, Altuner TK. Advances in echocardiography: Global longitudinal strain, intra-cardiac multidirectional flow imaging, and automated $3 \mathrm{~d}$ volume analysis. Heart Vessel Trans 2018;2:113e122.

22. Lang RM, Badano LP, Mor Avi V, Afilalo J, Armstrong A, Ernande L, et al. Guidelines and Standards Recommendations for Cardiac Chamber Quantification by Echocardiography in Adults: An Update from the American Society of Echocardiography and the European Association of Cardiovascular Imaging. Eur Heart J Cardiovasc Imaging 2015; 16:233e271.

23. Yeh ET, Bickford CL. Cardiovascular complications of cancer therapy:
Incidence, pathogenesis, diagnosis, and management. J Am Coll Cardiol 2009;53:2231-47.

24. Von Hoff DD, Layard MW, Basa P, Davis HL Jr., Von Hoff AL, Rozencweig M, et al. Risk factors for doxorubicin-induced congestive heart failure. Ann Intern Med 1979;91:710-7.7.

25. Motoki H, Koyama J, Nakazawa H, Aizawa K, Kasai H, Izawa A, et al. Torsion analysis in the early detection of anthracycline-mediated cardiomyopathy. Eur Heart J Cardiovasc Imaging 2012;13:95-103.

26. Cadeddu C, Piras A, Mantovani G, Deidda M, Dessì M, Madeddu C, et al. Protective effects of the angiotensin II receptor blocker telmisartan on epirubicin-induced inflammation, oxidative stress, and early ventricular impairment. Am Heart J 2010;160:487.e1-7.

27. Mantovani G, Madeddu $\mathrm{C}$, Cadeddu $\mathrm{C}$, Dessì M, Piras A, Massa E, et al. Persistence, up to 18 months of follow-up, of epirubicin-induced myocardial dysfunction detected early by serial tissue Doppler echocardiography: Correlation with inflammatory and oxidative stress markers. Oncologist 2008;13:1296-305.

28. Baratta S, Damiano M, Marchese M, Trucco J, Rizzo M, Bernok F, et al. Serum markers, conventional Doppler echocardiography, and two-dimensional systolic strain in the diagnosis of chemotherapy-induced myocardial toxicity. Rev Argent Cardiol 2013;81:151-8

29. Stoodley PW, Richards DA, Boyd A, Hui R, Harnett PR, Meikle SR, et al. Altered left ventricular longitudinal diastolic function correlates with reduced systolic function immediately after anthracycline chemotherapy. Eur Heart J Cardiovasc Imaging 2013;14:228-34.

30. Sawaya H, Sebag IA, Plana JC, Januzzi JL, Ky B, Tan TC, et al. Assessment of echocardiography and biomarkers for the extended prediction of cardiotoxicity in patients treated with anthracyclines, taxanes, and trastuzumab. Circ Cardiovasc Imaging 2012;5:596-603.

31. Thavendiranathan $\mathrm{P}$, Poulin F, Lim KD, Plana JC, Woo A, Marwick TH. Use of myocardial strain imaging by echocardiography for the early detection of cardiotoxicity in patients during and after cancer chemotherapy: A systematic review. J Am Coll Cardiol 2014;63:2751-68.

32. Kocabay G, Muraru D, Peluso D, Zucchini U, Mihaila S, Padayattil-Jose S, et al. Normal left ventricular mechanics by two-dimensional speckle-tracking echocardiography. Reference values in healthy adults. Rev EspCardiol (Engl Ed) 2014;67:651-8.

33. Takigiku K, Takeuchi M, Izumi C, Yuda S, Sakata K, Ohta N, et al. The Normal range of left ventricular 2-dimensional strain: Japanese ultrasound speckle tracking of the left ventricle (JUSTICE) study. Circ J 2012;76:2623-32.

34. Curigliano G, Lenihan D, Fradley M, Ganatra S, Barac A, Blaes A, et al. Management of cardiac disease in cancer patients throughout an oncological treatment: ESMO consensus recommendations. Ann Oncol 2020;31:171-90.

35. Zamorano JL, Lancellotti P, Rodriguez D, Aboyans V, Asteggiano R, Galderisi M, et al. 2016 ESC position paper on cancer treatments and cardiovascular toxicity developed under the auspices of the ESC committee for practice guidelines. Europ J Heart Failure 2016;19:9-42.

36. Alam S, Chandra S, Saran M, Chaudhary G, Sharma AM, Bhandhari M, et al. Indian Heart Journal 2019;71:468-75. 